\title{
Variação sazonal na abundância de Larus dominicanus (Aves, Laridae) no Saco da Fazenda, Itajaí, Santa Catarina
}

\author{
Luis Augusto Ebert ${ }^{1} \&$ Joaquim Olinto Branco ${ }^{2}$
}

\author{
1. Centro Universitário Leonardo da Vinci - UNIASSELVI, NEAD (Gestão Ambiental), Rodovia BR 470, km 71, nº 1040, 89130-000 \\ Indaial, Santa Catarina, Brasil. (luisaugustoebert@gmail.com) \\ 2. CTTMar, Universidade do Vale do Itajaí, Caixa Postal 360, 88301-970 Itajaí, Santa Catarina, Brasil. (branco@univali.br)
}

\begin{abstract}
Seasonal variation in the abundance of Larus dominicanus in the Saco da Fazenda, Itajaí, Santa Catarina, Brazil. The gull Larus dominicanus (Lichtenstein, 1823) is one of the most common coastal seabirds of the Brazilian coast, exploring several habitats and food types. The estuary known as Saco da Fazenda is an important feeding and resting area for the species. In this paper, we evaluate the population structure of L. dominicanus in this area. From February/2004 to January/2005, gulls were monitored in monthly census in Saco da Fazenda at intervals of two hours from 6:00 a.m. to 8:00 p.m. The fluctuations observed in the abundance of $L$. dominicanus along the year were significant, with the highest counts in March (181.6 \pm 35.1$)$ and the lowest in October $(21.0 \pm 4.9)$. Birds began occupation of the estuary in the first hours of the day, peaking at 2:00 p.m. (72.4 \pm 14.2$)$. In the end of the day the abundance reduced significantly with the lowest abundances at 8:00 p.m. (3.8 \pm 3.0$)$. Principal Components Analyses (PCA) revealed a positive correlation between the abundance of gulls and the temperature of the air. The fluctuations found in the abundance of $L$. dominicanus during this study can be correlated with different phases of the breeding cycle, while differences observed in the number of gulls along the day may be related to the activity of fishing fleet.
\end{abstract}

KEYWORDS. Kelp gull, population structure, fishing, Saco da Fazenda.

RESUMO. A gaivota Larus dominicanus (Lichtenstein, 1823) é uma das aves marinhas costeiras mais comuns do litoral brasileiro, sendo capaz de utilizar vários hábitats e explorar diferentes fontes alimentares. O estuário do Saco da Fazenda é uma importante área de forrageamento e descanso para a espécie. O objetivo deste trabalho foi o de avaliar a estrutura populacional de L. dominicanus no estuário. Durante o período de fevereiro/2004 a janeiro/2005, as gaivotas foram monitoradas mensalmente, com intervalos de duas horas entre os censos, das $6 \mathrm{~h}$ às $20 \mathrm{~h}$. As oscilações observadas na população ao longo do ano foram significativas, sendo as maiores contagens registradas em março $(181,6 \pm 35,1)$ e as menores em outubro $(21,0 \pm 4,9)$. A ocupação do estuário foi gradativa a partir das primeiras horas do dia, culminando com as maiores abundâncias às $14 \mathrm{~h}(72,4 \pm 14,2)$. Ao final do dia, o número de aves reduziu-se significativamente, com as menores abundâncias às $20 \mathrm{~h}(3,8 \pm 3,0)$. Através da Análise de Componentes Principais (ACP), foi possível estabelecer correlação positiva entre a abundância de gaivotas e a temperatura do ar. As oscilações encontradas na abundância de L. dominicanus durante o estudo podem ser atribuídas a eventos do ciclo de vida da espécie e as diferenças observadas no número de gaivotas ao longo do dia ao período de atividade da frota pesqueira.

PALAVRAS-CHAVE. Gaivota, estrutura populacional, pesca, Saco da Fazenda.

A gaivota Larus dominicanus (Lichtenstein, 1823) é uma das espécies de aves marinhas mais comuns ao longo da costa e das terras continentais adjacentes do Atlântico, ocorrendo do Brasil até a Terra do Fogo, e também na Península Antártica e ilhas subantárticas, litoral pacífico da América do Sul, sudoeste da África, Austrália e Nova Zelândia (SicK, 1997; YoRIO et al., 1998).

Essa espécie é considerada oportunista e generalista em seus hábitos de alimentação, sendo capaz de utilizar uma grande diversidade de presas (BRANCO, 2001) e explorar fontes antrópicas de alimento (GIACCARDI et al., 1997). Ocupa vários ambientes para nidificar, como ilhas, praias, dunas e lagoas costeiras (YoRIO et al., 1998), além de interagir com outras espécies de aves marinhas nesses ambientes (QUINTANA \& YORIO, 1998).

Expansões populacionais de gaivotas no mundo têm afetado negativamente outras espécies de aves costeiras, além de provocar conflitos em algumas cidades litorâneas, ao transportarem patógenos como Escherichia sp., Proteus sp. e Salmonela sp., capazes de causar enfermidades em humanos e animais domésticos (THOMAs, 1972; Furness \& Monaghan, 1987; Yorio et al., 1998).
No Brasil, não existem informações disponíveis sobre o tamanho das populações ou a magnitude dos distúrbios causados por L. dominicanus. Essa espécie é frequente nos levantamentos ornitológicos das regiões costeiras do sudeste e sul, mas apenas aspectos do ciclo de vida e reprodução foram estudados no litoral de Santa Catarina (SoAres \& Schiefler, 1995; Branco \& EBert, 2002; Branco, 2004). Este trabalho visa contribuir com informações sobre a espécie no Saco da Fazenda, Itajaí, Santa Catarina, que futuramente poderão ser utilizadas para o manejo ambiental com foco nos possíveis problemas que possam surgir em decorrência do eventual aumento na abundância de gaivotas.

\section{MATERIAL E MÉTODOS}

O Saco da Fazenda é parte integrante do estuário da foz do rio Itajaí-Açú, estando localizado a $26^{\circ} 55^{\prime} \mathrm{S}$ e $48^{\circ} 39^{\prime} \mathrm{W}$, no litoral centro-norte de Santa Catarina. Nessa área, foram realizados censos mensais da população de $L$. dominicanus durante o período de fevereiro de 2004 a 
janeiro de 2005. As contagens mensais foram efetuadas em intervalos de duas horas, entre $6 \mathrm{~h} \mathrm{e} 20 \mathrm{~h}$. O deslocamento pelo ecossistema foi efetuado a bordo de um barco a remo, o que permitiu boa aproximação das aves. A identificação das diferentes classes etárias (jovens, imaturos e adultos) foi realizada através da variação da cor da plumagem, da cor do bico e dos pés (Novelli, 1997; Sick, 1997), e a contagem das gaivotas através de observação direta dos exemplares com o auxílio de binóculo.

Após a padronização dos dados pela logaritmização das médias, foi aplicada a análise de variância “one-way" (SOKAL \& ROHLF, 1969), para verificar a existência de diferenças significativas entre as médias das classes etárias, bem como dos horários de censo e estações do ano. Também foi registrada a altura das marés, temperatura do ar e velocidade dos ventos, com o objetivo de estabelecer uma correlação entre essas variáveis e a abundância de gaivotas pela Análise de Componentes Principais (ACP).

\section{RESULTADOS}

Durante o período de estudo foram contadas 6.290 gaivotas. A maior abundância de jovens ocorreu em maio (média \pm desvio padrão $=30,3 \pm 5,9$ ind.), seguida de queda gradativa até agosto e ausência em setembro, com incremento a partir de outubro (Fig. 1). As maiores contagens de exemplares imaturos $(47,5 \pm 9,5)$ ocorreram em março, seguidas de redução até julho e ausência da classe em agosto e novembro, com pequeno incremento em setembro e flutuações até dezembro (Fig. 2). As gaivotas adultas apresentaram as maiores abundâncias em março $(103,7 \pm 19,8)$, com redução gradativa até julho, quando foram observados os menores contingentes (14,3 $\pm 3,0$; Fig. 3).

Ao analisar-se a abundância de $L$. dominicanus ao longo do ano e sem distinção entre as classes etárias (Fig. 4), verifica-se que ocorreram flutuações sazonais, com as maiores abundâncias médias em março (181,6 \pm $35,1)$, seguidas de queda acentuada em abril e oscilações a partir de maio até setembro, atingindo as menores abundâncias em outubro $(21,0 \pm 4,9)$.

A ANOVA $\left(\mathrm{F}_{11-84}=9,115 ; \mathrm{p}<0,001\right)$ ressaltou as variações na abundância da população ao longo do ano, onde a comparação entre as médias indica que essas estão relacionadas com as maiores contagens realizadas durante o mês de março. Apesar da aparente semelhança no padrão de flutuação anual das classes etárias (Figs. 1-3), ocorreram diferenças significativas $\left(\mathrm{F}_{2-33}=10,141 ; \mathrm{p}<0,001\right)$, ocasionadas pelas menores abundâncias de jovens em relação às demais classes de idade.

Quando confrontadas as abundâncias médias das classes por estação do ano, verifica-se que as diferenças observadas não foram significativas entre jovens, imaturos e adultos (Figs. 5-7). Porém, ao analisar-se a população sem discriminar a classe de idade (Fig. 8), ocorreram flutuações sazonais significativas $\left(\mathrm{F}_{3-28}=13,375 ; \mathrm{p}<0,001\right)$, influenciadas principalmente pelas menores abundâncias médias registradas durante os meses de primavera e inverno.

A abundância média das classes etárias aumentou gradativamente a partir das primeiras horas do dia até as $14 \mathrm{~h}$, seguida de redução moderada até atingir as menores frequências às 20 h (Figs. 9-11). Essas flutuações foram significativamente diferentes entre as gaivotas jovens $\left(\mathrm{F}_{7-88}=3,968 ; \mathrm{p}<0,001\right)$, imaturas $\left(\mathrm{F}_{7-88}=2,964 ; \mathrm{p}<0,001\right)$ e adultas $\left(\mathrm{F}_{7-88}=20,603 ; \mathrm{p}<0,001\right)$. Em uma análise geral da população (Fig. 12), constatase a mesma tendência de incremento na abundância média de gaivotas a partir das $6 \mathrm{~h}$, até alcançar as maiores contagens às 14 h $(72,4 \pm 14,2)$, seguidas de redução gradual até as $20 \mathrm{~h}$. O deslocamento progressivo das aves a partir das $14 \mathrm{~h}$ influenciou significativamente
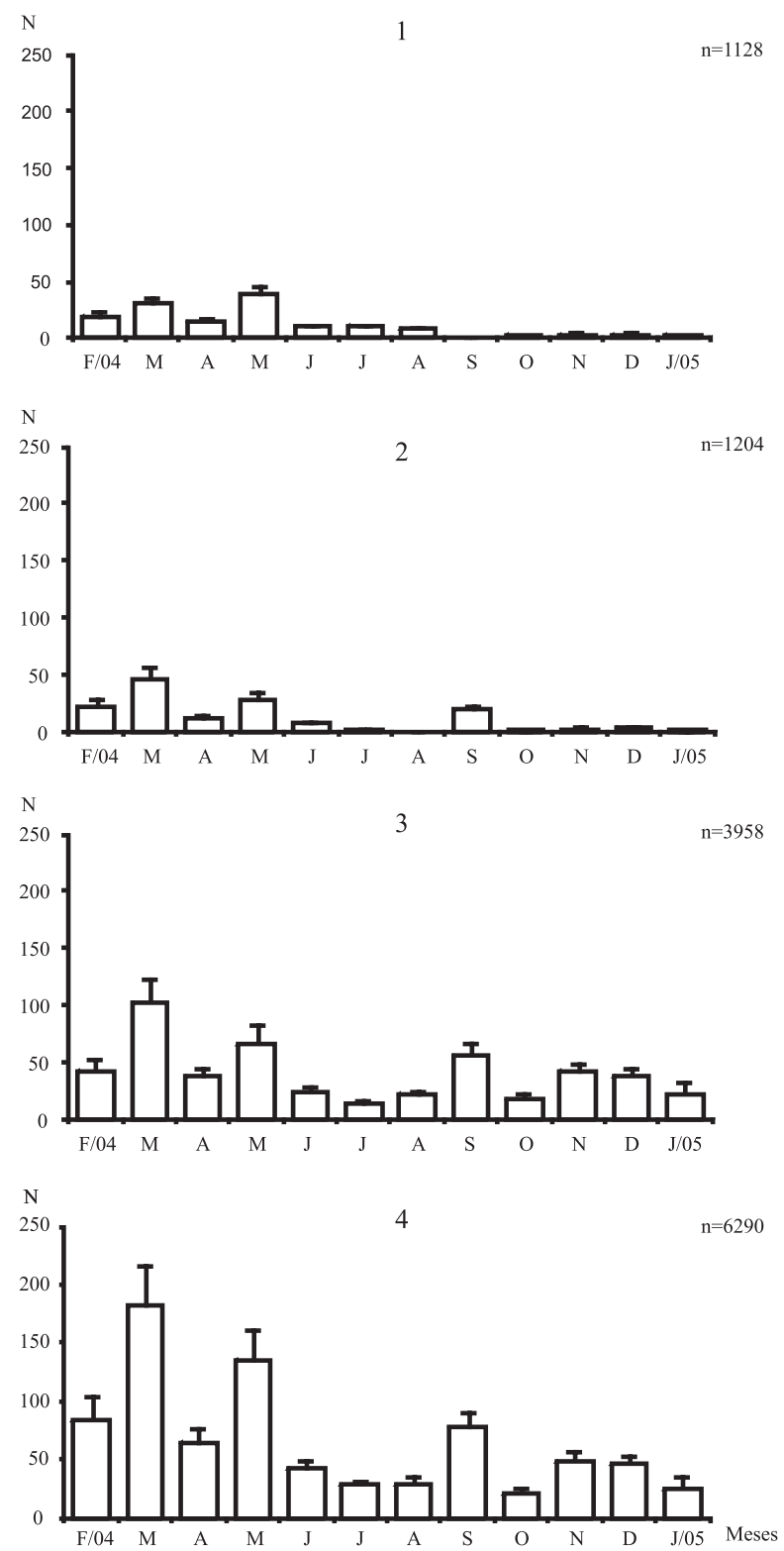

Figuras 1-4. Abundância média mensal (N) da população de Larus dominicanus (Lichtenstein, 1823) durante o período de fevereiro/ 2004 a janeiro/2005: 1, jovens; 2, imaturos; 3, adultos; 4, todas as idades agrupadas. 

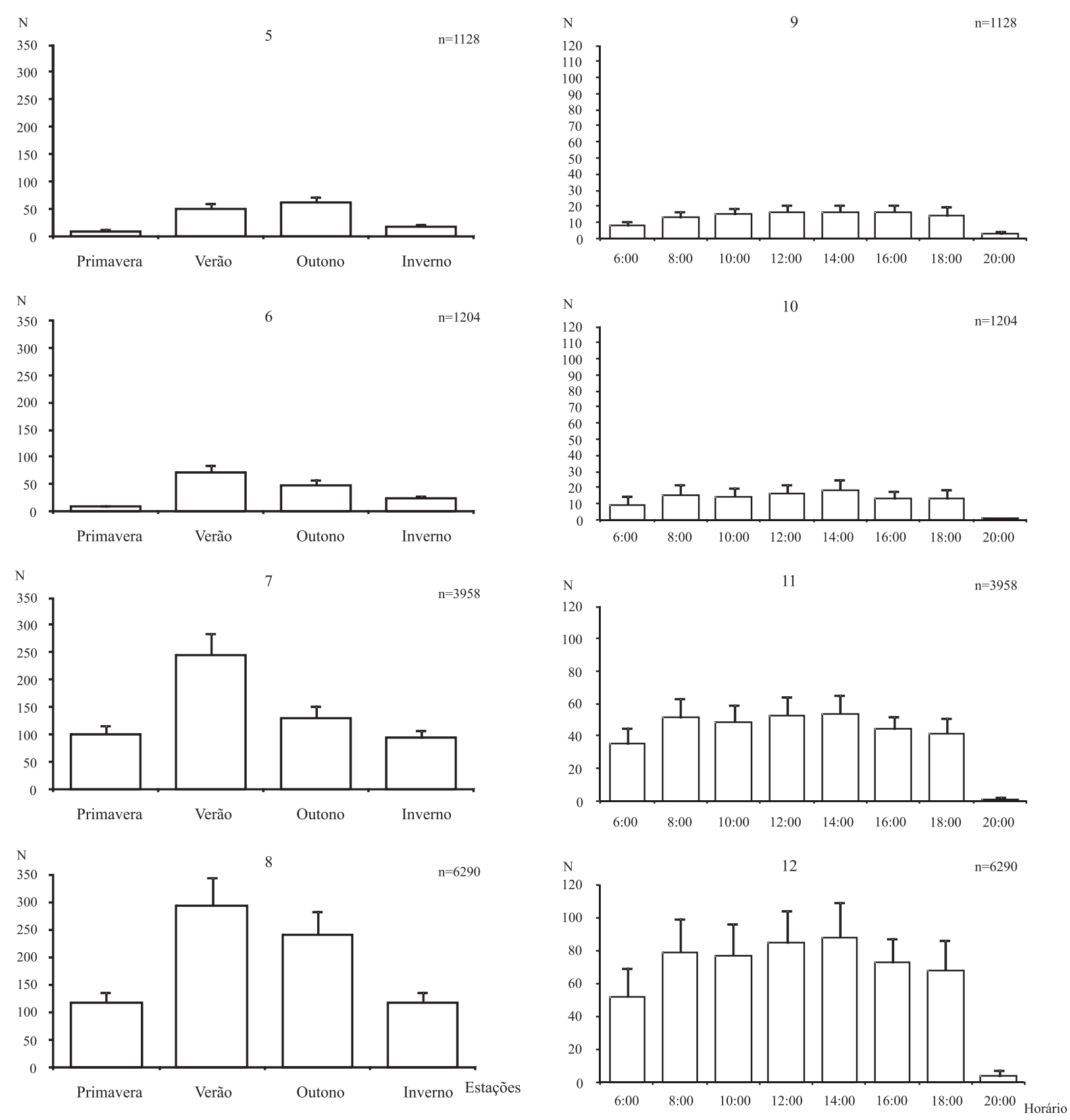

Figuras 5-8. Abundância média sazonal (N) da população de Larus dominicanus (Lichtenstein, 1823) durante o período de fevereiro/ 2004 a janeiro/2005: 5, jovens; 6 , imaturos; 7 , adultos; 8 , idades agrupadas.

a abundância de gaivotas no Saco da Fazenda $\left(\mathrm{F}_{7-88}=23,664 ; \mathrm{p}<0,001\right)$.

A Análise de Componentes Principais permitiu a extração de dois eixos fatoriais que explicaram $70 \%$ da variação dos dados (Fig. 13). O eixo I representou 40,8 \% dessa variação, indicando uma correlação positiva entre a abundância das gaivotas e a altura das marés, enquanto a velocidade dos ventos não esteve diretamente relacionada com a ocorrência das aves. O eixo II, com 29,7 \% da variação, esteve correlacionado positivamente com as maiores temperaturas e abundâncias de gaivotas (Fig. 13).

Figuras 9-12. Abundância média (N) da população de Larus dominicanus (Lichtenstein, 1823) durante o período de fevereiro/ 2004 a janeiro/2005: 9, jovens; 10, imaturos; 11, adultos; 12, idades agrupadas.

\section{DISCUSSÃO}

As flutuações na abundância de L. dominicanus ao longo do ano provavelmente estão associadas ao período reprodutivo, que abrange os meses de abril a dezembro (Bege \& PAuLi, 1988; SoARes \& SCHIEFler, 1995; Branco, 2004). Assim, as menores contagens de exemplares adultos entre junho a agosto são atribuídas ao deslocamento para as ilhas de nidificação, enquanto o incremento a partir de fevereiro indica o retorno dessas aves ao Saco da Fazenda e o ingresso de recrutas na população. Padrão semelhante de ocupação foi registrado 


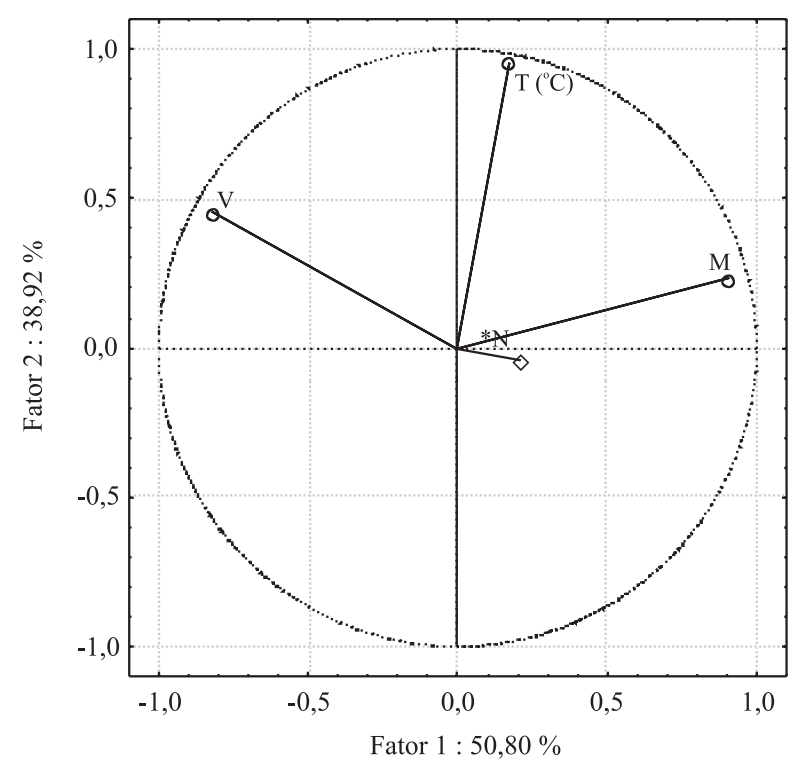

Figura 13. Eixos I e II extraídos a partir da Análise de Componentes Principais, aplicada sobre o número de exemplares de Larus dominicanus (Lichtenstein, 1823) (N), temperatura média do ar $\left(\mathrm{T}{ }^{\circ} \mathrm{C}\right)$, altura média de maré $(\mathrm{M})$ e velocidade média do vento $(\mathrm{V})$, durante o período de fevereiro/2004 a janeiro/2005.

em áreas de maricultura no litoral norte catarinense (BRANCO et al., 2001) e em depósitos de lixo na província de Chubut, na Patagônia argentina (YoRIo et al., 1998).

As variações sazonais nas classes etárias confirmam esse padrão de movimentação e ressaltam o recrutamento gradual de jovens, a partir da primavera, ao contingente do Saco da Fazenda, enquanto os picos de exemplares imaturos podem ser atribuídos à assincronia reprodutiva da espécie (SoARes \& Schiefler, 1995; Branco, 2004).

De maneira geral, as oscilações observadas na população ao longo do dia mantiveram um padrão definido, que demonstra a ocupação gradativa da área a partir das primeiras horas do dia, culminando com as maiores abundâncias às $14 \mathrm{~h}$. Esse comportamento está associado à utilização da ictiofauna descartada pela frota artesanal do camarão-sete-barbas no início da manhã, efetuado nas proximidades do Saco da Fazenda (BRAnco, 2000; 2001), e ao aproveitamento pelas gaivotas dos rejeitos de pesca oriundos das atividades de descarga de pescado nos portos de Itajaí e Navegantes, que ocorrem com maior frequência no período matutino.

A correlação positiva observada entre a abundância de gaivotas e a altura das marés, principalmente no início da tarde, pode estar associada às atividades de banho, manutenção da plumagem e descanso, enquanto o abandono da área no final da tarde sugere que as gaivotas estejam forrageando sobre os restos de peixes e resíduos orgânicos depositados nas margens do rio Itajaí-Açú e praias dos municípios de Itajaí e Navegantes (SCHIEFLER \& SoARES, 1994; Branco, 2000; BRANCO \& EBERT, 2002). Padrão semelhante de atividade foi registrado no litoral do Paraná, onde a presença de gaivotas consumindo peixes mortos nas praias foi frequente (MORAES \& KRUL, 1995). De acordo com GiACCARDI et al. (1997), o consumo de resíduos orgânicos durante a maré baixa pode ser atribuído à economia de energia gasta na captura de presas. Algumas observações indicam que ao anoitecer as gaivotas seguem para dormitórios em áreas afastadas do Saco da Fazenda, porém não existem dados conclusivos sobre os possíveis locais de repouso dessas aves.

$\mathrm{O}$ incremento nas populações de $L$. dominicanus registrado na costa oeste do Atlântico sul pode ser atribuído à flexibilidade da espécie no uso do hábitat e à utilização de fontes antrópicas de alimento. Essas características fazem das gaivotas boas competidoras por espaço e podem resultar em impactos negativos sobre outras espécies de aves costeiras através da predação, competição por espaço para nidificar e cleptoparasitismo. Além disso, o incremento das populações e suas atividades nas proximidades ou interior de cidades poderia resultar em conflitos com o homem, como a transmissão de patógenos (FRERE et al., 2000) e a ameaça de colisão com aeronaves. Embora o aeroporto de Navegantes esteja a 2 $\mathrm{km}$ de distância da área de estudo e a poucos metros da praia, ainda não há relatos de colisões, mas gaivotas afetando as atividades de transporte aéreo em alguns aeroportos da Argentina é um problema real (YORIO et al., 1998).

De acordo com Branco (2000), o Saco da Fazenda atua como importante área de descanso, manutenção da plumagem e alimentação para diferentes espécies de aves marinhas, apesar do impacto causado pelo afluxo de efluentes domésticos e resíduos sólidos. O presente estudo comprova a importância desse ambiente para a gaivota $L$. dominicanus em todos os quesitos citados acima pelo autor. No entanto, destaca-se a grande importância da oferta de alimentos oriundos das atividades de pesca artesanal e industrial provenientes dos barcos e empresas que operam nas proximidades do local estudado. Ficou comprovado ainda que a oscilação diária na abundância de gaivotas na área de estudo está relacionada com o período de alimentação dessas aves, enquanto que o deslocamento sazonal das gaivotas atribuiu-se ao período de reprodução.

O monitoramento da estrutura populacional de $L$. dominicanus ao longo dos anos poderá contribuir para a compreensão mais detalhada dos fatores que levam a essas oscilações na abundância da espécie, bem como das implicações dessas oscilações para a manutenção do equilíbrio ecológico e ambiental em um contexto regional.

\section{REFERÊNCIAS BIBLIOGRÁFICAS}

Bege, L. A. R. \& Pauli, B. T. 1988. As aves nas Ilhas Moleques do Sul - Santa Catarina: aspectos da ecologia, etologia e anilhameno de aves marinhas. Florianópolis, FATMA. $64 \mathrm{p}$.

Branco, J. O. 2000. Avifauna associada ao estuário do Saco da Fazenda. Revista Brasileira de Zoologia 17(2):387-394.

2001. Descartes da pesca do camarão sete-barbas como fonte de alimento para aves marinhas. Revista Brasileira de Zoologia 18(1):293-300.

(Org). 2004. Aves marinhas e insulares brasileiras: bioecologia e conservação. Itajaí, Univali. 266p.

Branco, J. O.; Braun, J. R. R. \& Verani, J. R. 2001. Seasonal variation in the abundance of seabirds in areas of mariculture. Brazilian Archives of Biology and Technology 44(4):395399.

Branco, J. O. \& Ebert, L. A. 2002. Estrutura da população de Larus dominicanus no estuário do Saco da Fazenda. Ararajuba 10(1):79-82. 
Frere, E.; Gandini, P. \& Martinez Peck, R. 2000. Gaivota cocinera (Larus dominicanus) como vector potencial de patógenos, en la costa Patagônica. Hornero 15:93-97.

Furness, R. W. \& Monaghan, P. 1987. Seabird ecology. London, Blackie. 432p.

Giaccardi, M.; Yorio, P. \& Lizurume, E. 1997. Patrones estacionales de la gaivota cocinera (Larus dominicanus) en un basural Patagónico y sus relaciones com el manejo de residuos urbanos y pesqueros. Ornitologia Neotropical 8:77-84.

Moraes, V. S. \& Krul, R. 1995. Aves associadas a ecossistemas de influência marítima no litoral do Paraná. Arquivos de Biologia e Tecnologia 38(1):121-134.

Novelli, R. 1997. Aves marinhas costeiras do Brasil (identificação e biologia). Porto Alegre, Editora Cinco Continentes. 90p.

Quintana, F. \& Yorio, P. 1998. Kelp gull Larus dominicanus predation on an imperial cormorant Phalacrocorax atriceps colony in Patagonia. Marine Ornithology 26:84,85.
Schiefler, A. F. \& Soares, M. 1994. Estudo comparativo da avifauna das praias de Navegantes e Laguna, Santa Catarina. Biotemas 7(1-2):31-45.

Sick, H. 1997. Ornitologia brasileira. Rio de Janeiro, Ed. Nova Fronteira. 912p.

Sodres, M. \& Schiefler, A. F. 1995. Reprodução de Larus dominicanus (AVES, Laridae), na Ilhota da Galeta, Laguna, SC, Brasil. Arquivos de Biologia e Tecnologia 38(1):313-316.

SoKal, R. R. \& RohlF, F. J. 1969. Biometry, the principles and practies of statistics in biological research. San Francisco, W. H. Freeman. 776p.

Tномаs, G. J. 1972. A review of gull damage and management methods at nature reserves. Biological Conservation 4:117127.

Yorio, P.; Bertelotti, M.; Gandini, P. \& Frere, E. 1998. Kelp Gulls Larus dominicanus breeding on the Argentina coast: population status and relationship with coastal management and conservation. Marine Ornithology 26:11-18.

Recebido em maio de 2007. Aceito em setembro de 2009. ISSN 0073-4721

Artigo disponível em: www.scielo.br/isz 